\title{
Impact of human papillomavirus genotype on response to treatment and survival in patients receiving radiotherapy for squamous cell carcinoma of the cervix
}

\author{
JANNATUL FERDOUSI ${ }^{1}$, YUTAKA NAGAI ${ }^{1}$, TSUYOSHI ASATO ${ }^{2}$, MAKOTO HIRAKAWA ${ }^{1}$, \\ MORIHIKO INAMINE ${ }^{1}$, WATARU KUDAKA ${ }^{1}$, KEN-ICHI KARIYA ${ }^{2}$ and YOICHI AOKI ${ }^{1}$ \\ ${ }^{1}$ Department of Obstetrics and Gynecology, Faculty of Medicine; ${ }^{2}$ Division of Cell Biology, \\ Graduate School of Medicine, University of the Ryukyus, Okinawa 903-0215, Japan
}

Received January 7, 2010; Accepted March 15, 2010

DOI: 10.3892/etm_00000083

\begin{abstract}
To determine the clinical implications and prognostic value of the human papillomavirus (HPV) genotype, we evaluated the various HPV types in patients receiving radiotherapy for squamous cell carcinoma of the cervix. The study population included 113 invasive squamous cell carcinoma patients treated with radiation or chemoradiation between 1993 and 2002. The median age of the patients was 61 years. Tumors were classified by the International Federation of Gynecology and Obstetrics staging as stage IB in 11 patients, stage II in 39, stage III in 57 and stage IVA in 6 patients. To investigate HPV infection and its genotypes in the tumor specimens, L1 consensus PCR was performed followed by the direct nucleotide sequencing of the PCR products. Ninety-five samples $(84.1 \%)$ were positive for HPV DNA. The most prevalent type was HPV-16 (34.7\%). Poorer response to radiotherapy was observed in the patients with the HPV-16 genotype, in which 7 of the 33 patients had persistent disease. Only 1 of the 10 patients with HPV-58, 1 of the 5 with HPV-31 and 5 of the 10 patients with HPV-33 had a recurrence. The 5-year survival rate was 90, 80, 69.4 and $39 \%$ in the HPV-58, HPV-31, HPV-16 and HPV-33 type groups, respectively. Patients with HPV-31 and HPV-58 types were found to have better survival, whereas patients with the HPV-33 type experienced a higher risk of death. HPV genotyping may serve as a potential biomarker of response to radiation and prognosis in cervical carcinoma patients undergoing radio- or chemoradiotherapy.
\end{abstract}

Correspondence to: Dr Yoichi Aoki, Department of Obstetrics and Gynecology, Faculty of Medicine, University of the Ryukyus, 207 Uehara Nishihara, Okinawa 903-0215, Japan

E-mail: yoichi@med.u-ryukyu.ac.jp

Key words: human papillomavirus genotype, cervical cancer, radiotherapy, chemoradiotherapy, radiation response, prognosis

\section{Introduction}

Cervical cancer is the second most common cancer among Japanese women. Studies in several parts of the world have demonstrated a very strong association between the human papillomavirus (HPV) and cervical cancer with odds ratios of over 15 (1). Prospective studies have shown that infection with high-risk HPV precedes the development of cervical neoplasia (2). HPV has now been accepted as a necessary cause of cervical cancer (3).

It has been accepted that the prognosis of cervical carcinoma is related to clinical stage, lymph node metastasis, parametrial invasion, primary tumor size, histological type, depth of cervical stromal invasion and lymph vascular space involvement. (4) However, recurrent cervical carcinoma will develop in approximately $10-15 \%$ of stage I-IIA patients and in $30-50 \%$ of stage IIB-III patients due to variable responses to surgery or radiotherapy (5). Further stratification based on novel specific molecular markers for cervical carcinoma is crucial to improve the survival rate in this heterogeneous group of patients. This type of classification may help clinicians in developing appropriate therapeutic strategies for molecular subtyping of patients at a high risk of disease recurrence.

Although HPV infection has been established as an important initial event in the tumorigenesis of cervical carcinoma, reports on the clinical impact of different HPV types are conflicting. Previous attempts to determine the prognostic significance of the presence or absence of detectable HPV DNA and HPV types in cervical cancer patients have generated conflicting results (6-13). Some studies have reported that patients infected with HPV-18 had worse prognoses and higher disease recurrence rates than patients with HPV-16 infection $(6-8,10)$. In a recent study, infection with multiple HPV types was also considered an indicator of poor response to radiotherapy $(7,11)$. However, other reports did not demonstrate an association between HPV type and clinical outcome $(8,9,12,13)$. Conversely, Lai et al reported that cervical carcinoma patients infected with HPV-58-related types had a favorable outcome (10). These discrepancies have made it difficult to interpret the relevance of the HPV genotype and clinical outcome. 
The present study was designed to analyze the relationship between HPV DNA status and clinicopathological parameters in order to further elucidate the role of the HPV type in relation to clinical outcome of cervical carcinoma. Finally, to determine the clinical implications and prognostic value of the HPV genotype in cervical carcinoma, we evaluated whether various HPV types in patients receiving radiotherapy for squamous cell carcinoma of the cervix correlate with survival.

\section{Materials and methods}

Patients. The study population included 113 invasive squamous cell carcinoma patients treated with radiation or chemoradiation between 1993 and 2002. These patients were successfully followed-up at the University of the Ryukyus Hospital, Okinawa, Japan. Patient age, International Federation of Gynecology and Obstetrics (FIGO) stage, tumor size, hemoglobin level before treatment and status of lymph node enlargement were recorded after thorough clinical investigations. Written informed consent was obtained from all patients, and our Institutional Research Board approved the study.

Radiotherapy and concurrent chemoradiotherapy. The patients were treated with anterior-posterior and posteroanterior parallel opposed ports of external beam radiotherapy (EBRT). The dose of ERBT was $50 \mathrm{~Gy}$ delivered in 25 fractions. The center shield (4-cm width at the midline) was set up after delivering $40 \mathrm{~Gy}$. High-dose rate intracavitary radiotherapy was delivered once a week with a fraction dose of $6 \mathrm{~Gy}$ at point A for 3 or 4 times. Forty patients received cisplatin $\left(20 \mathrm{mg} / \mathrm{m}^{2}\right)$ for 5 days every 3 weeks, concomitant with radiotherapy (14).

Typing of HPV DNA. Specimens were freshly collected from biopsies. They were snap frozen in liquid nitrogen and stored at $-70^{\circ} \mathrm{C}$ until use. Part of each specimen was examined pathologically for diagnosis. To test for the presence of HPV, the DNA extracted from the specimen was subjected to polymerase chain reaction (PCR), using an L1 consensus primer pair [L1C1 and L1C2; reported by Yoshikawa et al (15)], as described elsewhere (16). PCR with a $\beta$-globin primer pair was performed in parallel, and $\beta$-globin-negative samples were not included in further analyses. The L1 PCR products obtained from HPV-positive samples were stored frozen. To identify the HPV genotypes, direct nucleotide sequencing of PCR products was performed as described previously (27). Similarities of the obtained L1 sequences between those of various HPV DNA sequences in the database were examined with BLAST analysis (http://www.ncbi.nih.gov/BLAST).

Statistical analysis. JMP 6.0 software (SAS Institute, Cary, NC) was used for statistical analyses. Survival curves were estimated by the Kaplan-Meier method, and differences were tested by the log-rank test. Furthermore, p-values $<0.05$ were considered significant.

\section{Results}

Patient characteristics are listed in Table I. The median age of the patients was 61 years (range 30-80 years). Tumors were
Table I. Patient characteristics.

\begin{tabular}{|c|c|c|}
\hline Variables & No. $(n=113)$ & $\%$ \\
\hline \multicolumn{3}{|l|}{$\begin{array}{l}\text { Age (median } 61 \text { years, } \\
\text { range } 30-80 \text { years) }\end{array}$} \\
\hline$\leq 50$ & 34 & 30.1 \\
\hline$>50$ & 79 & 69.9 \\
\hline \multicolumn{3}{|l|}{ FIGO stage } \\
\hline $\mathrm{I}$ & 11 & 9.7 \\
\hline II & 39 & 34.5 \\
\hline III & 57 & 50.4 \\
\hline IV & 6 & 4.4 \\
\hline \multicolumn{3}{|l|}{ Tumor size } \\
\hline$\leq 4 \mathrm{~cm}$ & 61 & 54.0 \\
\hline$>4 \mathrm{~cm}$ & 52 & 46.0 \\
\hline \multicolumn{3}{|l|}{ Lymph node enlargement } \\
\hline Positive & 53 & 46.9 \\
\hline Negative & 60 & 53.1 \\
\hline \multicolumn{3}{|l|}{ Hemoglobin level } \\
\hline$\leq 11.3$ & 42 & 37.2 \\
\hline$>11.3$ & 71 & 62.8 \\
\hline \multicolumn{3}{|l|}{ Treatment } \\
\hline Chemoradiotherapy & 40 & 35.4 \\
\hline Radiotherapy alone & 73 & 64.6 \\
\hline \multicolumn{3}{|l|}{ Response to treatment } \\
\hline Persistence & 16 & 23.0 \\
\hline Complete response & 97 & 77.0 \\
\hline \multicolumn{3}{|l|}{ Prognosis } \\
\hline No evidence of disease & 69 & 61.1 \\
\hline Died of disease & 44 & 38.9 \\
\hline
\end{tabular}

classified by FIGO staging as stage IB in 11 patients, stage II in 39, stage III in 57 and stage IVA in 6 patients. The tumor size of the cervix, determined by magnetic resonance imaging (MRI) was $\leq 4 \mathrm{~cm}$ in 50 cases and $>4 \mathrm{~cm}$ in the remaining 53 cases. Pelvic lymph node enlargement, which was defined as an enlargement $>10 \mathrm{~mm}$ in the shortest dimension by computed tomography or MRI, was observed in 53 cases. Follow-up examinations were conducted every month for the first year, every other month for the second year and then every 3-6 months. During the follow-up period (median 64 months; range 6-164 months), 45 patients died, 44 deaths being directly related to the disease.

Prevalence and the HPV genotypes in cervical carcinoma are summarized in Table II. Of the 113 specimens, $95(84.1 \%)$ were positive for HPV DNA. The most prevalent types were HPV-16 (34.7\%), HPV-33 (10.5\%), HPV-58 (10.5\%) and HPV-52 (7.3\%). Multiple HPV infections (HPV-16 and HPV-33) were detected in only 1 sample. The relationship between HPV genotypes and clinicopathological variables are shown in Table III. The HPV genotype was found to be associated with age. HPV-16 type was found more frequently in younger patients (16 of 33 cases) as compared to other HPV 
Table II. HPV genotype distribution.

\begin{tabular}{lrr}
\hline Genotype & No. & $\%$ \\
\hline Total & 113 & \\
HPV(+) & 95 & 84.1 \\
HPV-16 & 33 & 34.7 \\
HPV-18 & 3 & 3.2 \\
HPV-31 & 5 & 5.2 \\
HPV-33 & 10 & 10.5 \\
HPV-35 & 3 & 3.2 \\
HPV-51 & 1 & 1.1 \\
HPV-52 & 7 & 7.3 \\
HPV-53 & 3 & 3.2 \\
HPV-54 & 1 & 1.1 \\
HPV-56 & 4 & 4.2 \\
HPV-58 & 10 & 10.5 \\
HPV-59 & 3 & 3.2 \\
HPV-66 & 2 & 2.1 \\
HPV-70 & 1 & 1.1 \\
HPV-73 & 1 & 1.1 \\
HPV-82 & 1 & 1.1 \\
HPV-16 +33 & 1 & 1.1 \\
Undetermined & 6.4 \\
HPV(-) & & 15.9 \\
\hline
\end{tabular}

types. However, the HPV genotype was not associated with other clinicopathological parameters, namely, FIGO stage, lymph node swelling, tumor size and hemoglobin level.

Poorer response to radiotherapy was observed in the HPV-16 genotype. Although 53 of the 56 patients with other types of HPV achieved complete response to radiotherapy, 7 of the 33 patients with HPV-16 had persistent disease after completion of radiotherapy $(p=0.096)$. In terms of disease recurrence, among patients with HPV-16, 11 had a local recurrence. Only 1 of the 10 patients with HPV-58, 1 of the 5 patients with HPV-31 and 5 of the 10 patients with HPV-33 had a recurrence. Forty-four patients (39.2\%) died of cervical carcinoma at the end of the follow-up period. For overall survival, univariate analysis based on the log-rank test is summarized in Table IV. The 5-year survival rate was $90 \%$ in the HPV-58 type group ( $\mathrm{n}=10), 80 \%$ in the HPV-31 type group $(n=5), 69.4 \%$ in the HPV-16 type group $(n=33)$ and $39 \%$ in the HPV-33 type group $(n=10)$. Patients with HPV-31 and HPV-58 types were found to have better survival than patients with the HPV-16 type (Fig. 1), which was not statistically significant. However, patients with the HPV-33 type experienced a higher risk of death than patients with the HPV-58 ( $\mathrm{p}=0.0508)$, HPV-31 ( $p=0.3298)$ and HPV-16 type ( $p=0.4726$; Fig. 1$)$.

\section{Discussion}

In this study, we report the clinicopathologic factors of 113 squamous cell carcinoma patients treated with radio- or chemoradiotherapy, and the results of our analysis of these factors with regard to HPV genotypes. Previous attempts

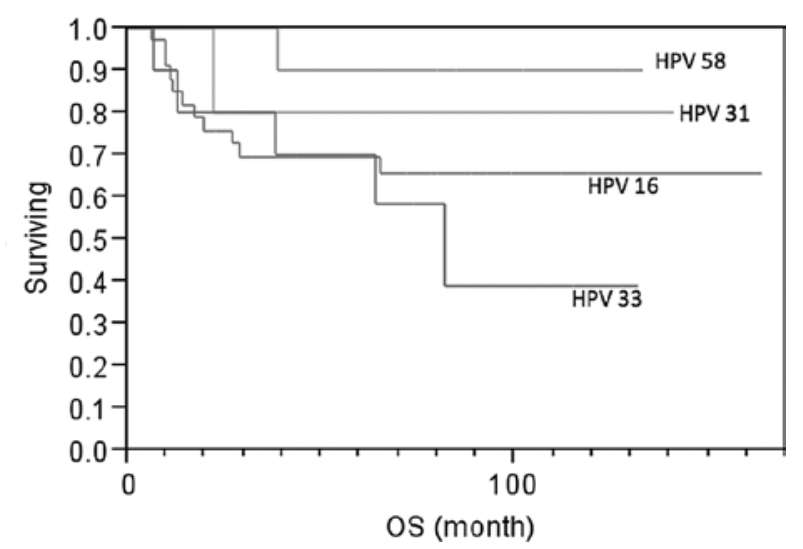

Figure 1. Kaplan-Meier curves for overall survival stratified in accordance with human papillomavirus (HPV)-16, HPV-31, HPV-33 and HPV-58 in cervical carcinoma patients.

to determine the prognostic significance of the presence or absence of detectable HPV DNA and HPV types in cervical cancer patients have generated conflicting results (6-13). These discrepancies have made it difficult to interpret the relevance of the HPV genotype and clinical outcome.

We sought to identify the prognostic significance of the HPV DNA genotype only in squamous cell carcinoma patients treated with radio- or chemoradiotherapy. A poorer response to radiotherapy was observed in the HPV-16 genotype. Although 53 of the 56 patients with other types of HPV achieved complete response to radiotherapy, 7 of the 33 patients with HPV-16 had persistent disease after completion of radiotherapy. We are not aware of such a report in the literature. Some biological difference may exist between HPV-16 and other types of HPV-positive cancer cells; we need to further investigate this using a larger population of patients.

Results of prognostic analysis showed that the HPV-58 and HPV-31 types had a tendency to predict favorable survival, thus suggesting that these 2 types may be predictors of good prognosis. Although this finding has to be substantiated in a larger number of patients, HPV genotyping has the potential to serve as a biomarker of prognosis in combination with established markers in patients with squamous cell carcinoma of the cervix.

Despite the high prevalence of HPV-58 and its related types in East Asia, the clinical behavior and prognostic value of these viral infections are unclear. The present study showed that HPV-31 and HPV-58 were more prevalent in the older age group than in the younger. Increasing prevalence of HPV types other than 16 and 18 was observed in older patients in Japan (16-18), where HPV-58 was also relatively prevalent. Patients infected with HPV-31 and HPV-58 may actually experience an indolent clinical course and develop cancer at an older age, since it was found that they were not older than other patients with squamous cell intraepithelial lesions without such infection (16). Infection by HPV-31 and HPV-58 may be partly responsible for cervical cancer in the older population in these areas. Lai et al (10) demonstrated that, in comparison to the HPV-16-related group, the relative risk of death in the HPV-58-related (types 58, 33 and 52) group was 0.32 (95\% CI 0.07-1.49); these types were prevalent in the older population and appeared to confer a favorable prognosis. Huang et al (11) 


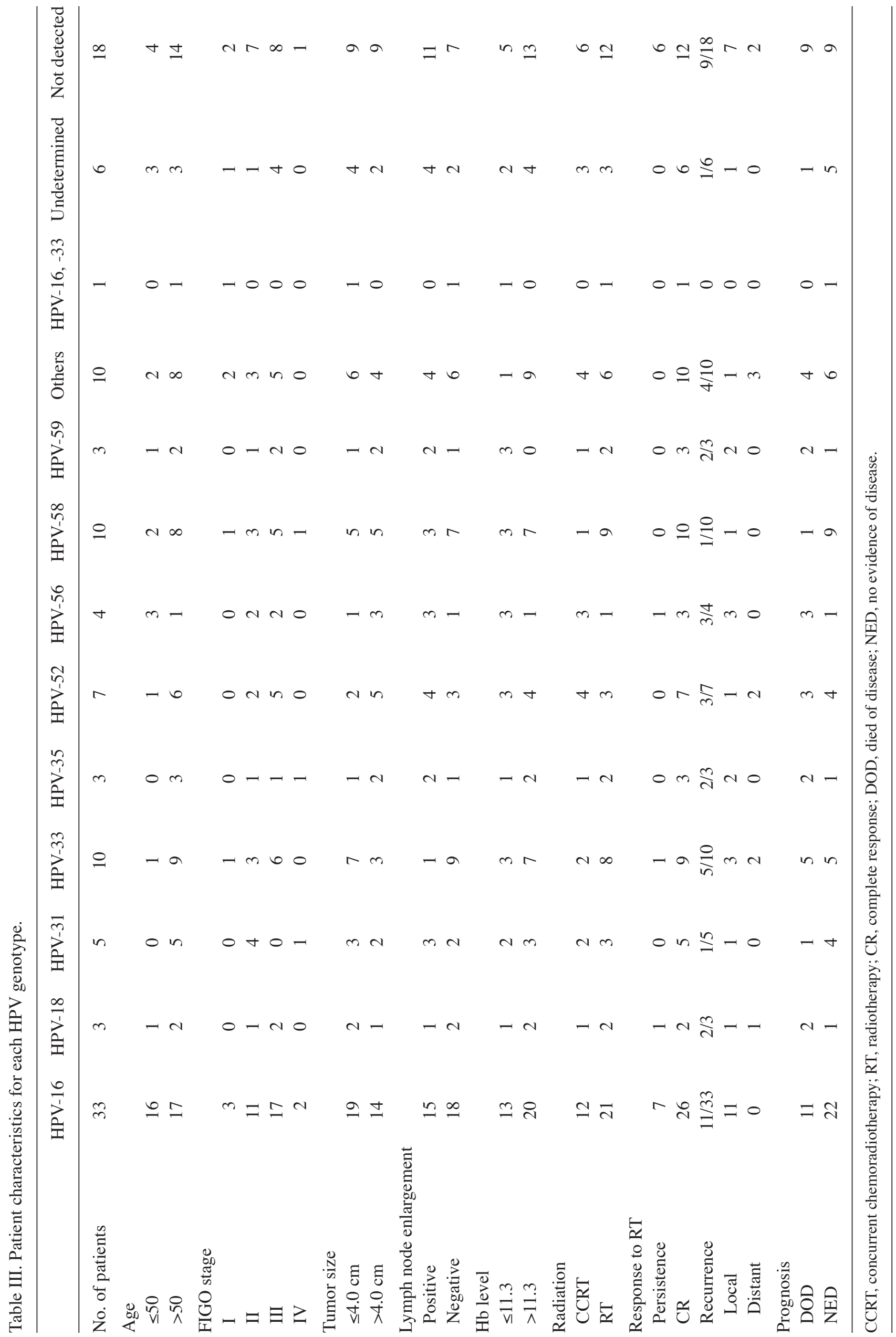


Table IV. Life-table analysis of 113 patients with cervical cancer.

\begin{tabular}{lcc}
\hline Characteristics & $\begin{array}{c}\text { No. of } \\
\text { patients }\end{array}$ & $\begin{array}{c}5 \text {-year overall } \\
\text { survival }(\%)\end{array}$
\end{tabular}

\section{Age}

$\leq 50$

$>50$

34

79

57.6

69.1

FIGO stage

I-II

III-IV

50

79.0

44.7

Tumor size

$\leq 4 \mathrm{~cm}$

$>4 \mathrm{~cm}$

61

71.0

0.140

Lymph node enlargement

\section{Positive}

Negative

53

60

41.6

86.5

Hemoglobin level

\begin{tabular}{lrcc}
$\leq 11.3$ & 42 & 44.6 & $<0.001$ \\
$>11.3$ & 71 & 77.4 & \\
HPV type $^{\mathrm{a}}$ & & & \\
16 & 33 & 69.4 & vs. HPV16 \\
18 & 3 & 33.0 & 0.23 \\
31 & 5 & 80.0 & 0.50 \\
33 & 10 & 39.0 & 0.47 \\
35 & 3 & 33.0 & 0.42 \\
52 & 6 & 68.0 & 0.51 \\
56 & 4 & 25.0 & 0.07 \\
58 & 10 & 90.0 & 0.14 \\
59 & 3 & 67.0 & 0.67 \\
Others & 10 & 60.0 & 0.89 \\
Undetermined & 6 & 83.0 & 0.30 \\
\hline
\end{tabular}

${ }^{\mathrm{a} O n e}$ case with HPV-16 + 33 was excluded.

showed that the presence of HPV-31-related types was an independent predictor of better survival in patients with cervical carcinoma. However, in their study, HPV-31-related types included types $31,33,35$ or 67 , and hence, the results of these two studies are not exactly consistent with our findings. If the conflicting results are due to misclassification and the number of HPV cases, analyses of a larger number of patients with stratification by distinct type would be useful to clarify the discrepancies. Further investigation of the natural history of HPV-31- and HPV-58-associated cervical neoplasia and their underlying biological mechanisms are obviously warranted.

On the other hand, patients with the HPV-33 type experienced higher risk of death than patients with the HPV-16 type, which had a relatively poor response to radiotherapy and a significantly worse prognosis than patients with HPV-58 or HPV-31. With regard to HPV-33 in particular, the patients had more favorable clinicopathological factors, such as prevalence in older age, smaller tumor size, less frequent lymph node swelling and good response to radiotherapy; however, a higher rate of distant recurrence and poor prognosis were observed. Hagmar et al (19) found that patients with HPV-33- or HPV-18associated tumors had worse prognoses than patients with other types of HPV infections, although the reason for this remains unclear. It is necessary to investigate the underlying biological mechanisms of HPV-33-associated cervical cancer.

Previous studies have reported that patients with HPV-18containing tumors have an increased risk of death and disease recurrence. On the molecular level, strong evidence suggests that HPV-18 confers increased oncogenic potential, given the fact that its transforming activity was 5 times that of HPV-16 in cell culture systems (20). This finding is consistent with the findings of previous studies $(8,9,11,12)$. Unfortunately, we were unable to confirm this since only 3 patients had HPV-18. Other groups included HPV-52, HPV-53, HPV-54, HPV-66, HPV-70, HPV-73 and HPV-82, which were all classified as intermediate- or low-risk subtypes for carcinogenesis of cervical cancer. Further investigation on the maintenance of malignant phenotypes by these intermediate- or low-risk HPV subtypes is also warranted.

The results of the present study demonstrated the possibility that HPV-58 and HPV-31 are predictors of good prognosis, HPV-33 is a predictor of poor prognosis and the HPV-16 type is a predictor of poor response in squamous cell carcinoma patients treated with radiation or chemoradiation. Although this finding must be substantiated in a larger number of patients, HPV genotyping may serve as a potential biomarker of prognosis in combination with established markers in patients with cervical carcinoma.

\section{References}

1. Bosch FX, Manos MM, Munoz N, et al: Prevalence of human papillomavirus in cervical cancer: a worldwide perspective. International Biological Study on Cervical Cancer (IBSCC) Study Group. J Natl Cancer Inst 87: 796-802, 1995.

2. Liaw KL, Glass AG, Manos MM, et al: Detection of human papillomavirus DNA in cytologically normal women and subsequent cervical squamous intraepithelial lesions. J Natl Cancer Inst 91: 954-960, 1999.

3. Walboomers JM, Jacobs MV, Manos MM, et al: Human papillomavirus is a necessary cause of invasive cancer worldwide. $\mathrm{J}$ Pathol 189: 12-19, 1999.

4. Kosary CL: FIGO stage, histology, histologic grade, age and race as prognostic factors in determining survival for cancers of the female gynecological system: an analysis of 1973-87 SEER cases of cancers of the endometrium, cervix, ovary, vulva and vagina. Semin Surg Oncol 10: 31-46, 1994.

5. Coia L, Won M, Lanciano R, et al: The patterns of care outcome study for cancer of the uterine cervix. Results of the Second National Practice Survey. Cancer 66: 2451-2456, 1990.

6. Schwartz SM, Daling JR, Shera KA, et al: Human papillomavirus and prognosis of invasive cervical cancer: a population-based study. J Clin Oncol 19: 1906-1915, 2001.

7. Bachtiary B, Obermair A, Dreier B, et al: Impact of multiple HPV infection on response to treatment and survival in patients receiving radical radiotherapy for cervical cancer. Int $\mathbf{J}$ Cancer 102: 237-243, 2002.

8. Kristensen GB, Karlsen F, Jenkins A, et al: Human papilloma virus has no prognostic significance in cervical carcinoma. Eur J Cancer 32A: 1349-1353, 1996.

9. Van Bommel PF, van den Brule AJ, Helmerhorst TJ, et al: HPV DNA presence and HPV genotypes as prognostic factors in low-stage squamous cell cervical cancer. Gynecol Oncol 48: 333-337, 1993.

10. Lai HC, Sun CA, Yu MH, et al: Favorable clinical outcome of cervical cancers infected with human papilloma virus type 58 and related types. Int J Cancer 84: 553-557, 1999. 
11. Huang LW, Chao SL and Hwang JL: Human papillomavirus31-related types predict better survival in cervical carcinoma. Cancer 100: 327-334, 2004.

12. Graflund M, Sorbe B, Sigurdardottir S, et al: HPV-DNA, vascular space invasion, and their impact on the clinical outcome in earlystage cervical carcinomas. Int J Gynecol Cancer 14: 896-902, 2004.

13. Tong SY, Lee YS, Park JS, et al: Papillomavirus genotype as a prognostic factor in carcinoma of the uterine cervix. Int J Gynecol Cancer 17: 1307-1313, 2007.

14. Hirakawa M, Nagai Y, Inamine M, et al: Predictive factors of distant recurrence in locally advanced squamous cell carcinoma of the cervix treated with concurrent chemoradiotherapy. Gynecol Oncol 108: 126-129, 2008.

15. Yoshikawa H, Kawana T, Kitagawa K, et al: Detection and typing of multiple genital human papillomaviruses by DNA amplification with consensus primers. Jpn J Cancer Res 82: 524-531, 1991.
16. Asato T, Maehama T, Nagai Y, et al: A large case-control study on cervical cancer risk of HPV infection in Japan by nucleotide sequencing-based genotyping. J Infect Dis 189: 1829-1832, 2004.

17. Inoue M, Sakaguchi J, Sasagawa $\mathrm{T}$, et al: The evaluation of human papillomavirus DNA testing in primary screening for cervical lesions in a large Japanese population. Int J Gynecol Cancer 16: 1007-1013, 2006.

18. Miura S, Matsumoto K, Oki A, et al: Do we need a different strategy for HPV screening and vaccination in East Asia? Int J Cancer 119: 2713-2715, 2006.

19. Hagmar B, Christensen JJ, Johansson B, et al: Implications of human papillomavirus type for survival in cervical squamous cell carcinoma. Int J Gynecol Cancer 5: 341-345, 1995.

20. Barbosa MS and Schlegel R: The E6 and E7 genes of HPV-18 are sufficient for inducing two-stage in vitro transformation of human keratinocytes. Oncogene 4: 1529-1532, 1989. 